\title{
A Study on the Para-social Interaction Between Idols and Fans in Virtual Applications Case Study of Lysn Bubble
}

\author{
Yixuan Zhang
}

Duke Kunshan University

yz521@duke.edu

\begin{abstract}
Advances in media technology have accelerated the development of social media, and also assist the evolution of idol industry. This paper delves into the para-social interaction in the virtual application - Lysn Bubble through the methodology of participatory observation and text analysis. From these three perspectives: behind-the-scene content, simulated private chatting environment and establishment of membership communities, para-social interaction is well-built between fans and idols. Bubble meets the fans' requirements for intimate relationships, breaks through the previous barrier of two-way communication between idols and fans, and introduces the concept of purchasing information and communication with idols into para-social interaction.
\end{abstract}

Keywords: para-social interaction, fandom culture, cybervigilantism, virtual application, K-pop idol.

\section{INTRODUCTION}

With the development of the Internet, social media has brought about great changes in the society, affecting people's daily life, and helped the rapid development of the idol industry. The idol industry is using social media to build new models of idol-fan interaction. In February 2020, S.M. Entertainment launched an interactive app called DearU Bubble on its Lysn platform, where fans pay a certain amount of money for tickets of specific idols to receive unique private images, text and videos sent by them. Bubble's sales during the second quarter of last year reportedly reached 4.2 billion won. Unlike traditional social platforms, Bubble involves money cost in the interaction between idols and fans, providing a new way of thinking about this para-social interaction.

On the basis of literature analysis, this paper adopts the research methods of status quo analysis and self-participation observation to study the para-social interaction in Bubble. Para-social interaction will first be built in this application from three perspectives. Then, this paper will analyze the new characteristics of para-social interaction based on these three perspectives.

\section{LITERATURE REVIEW}

There is a considerable amount of literature on the para-social interaction and such interaction between idols and fans. According to Horton and Wohl, para-social interactions provide audiences with the illusion of having face-to-face communication with celebrities. People consider themselves as knowing celebrities personally while don't have any relationship in actual. Traditional para-social interaction usually takes place in media conditions such as television, radio and theater stage[4]. Under such circumstances of traditional media such as paper media and television, people are more likely to passively perceive and it is impossible for celebrities to feel the audience through the media simultaneously despite space and time. With generally a large number of fans, celebrities arouse the attention of them through some media behaviors, including performances and other activities, so that fans can have a certain emotional connection with them.

However, following the popularity of internet, social media platforms like Twitter and Facebook have then become valuable channels for enhancing relationships between celebrities and their fans[1]. New media and traditional media environment show the same interaction phenomenon, that is, celebrities have a large number of fans, and attract fans' attention through 
online and offline media behaviors. Due to the differences in their own communication environment, para-social interaction also changes in the new media environment, from only the one-sided intimacy of the audience, to the preliminary forming of two-way interaction[11]. The single-way interaction used to happen in traditional media, while now fans are reposting, commenting or sending private messages when following their idols' accounts on social media platforms, and sometimes idols may reply to only a few of chosen individuals. Study also indicates that the more celebrities share with fans, the higher degree which fans engage in para-social relationships are[6]. Along with the development of idol industry and fandom culture, there appears a trend of idols catering to fans in reverse, such as having lottery, fan-signing and other forms of events. These behaviors all promote the para-social interaction to some extent, thus stimulating the development of fan economy.

The above papers give a general idea of how para-social interactions play roles between idols and their fans. In 2021, Zhu put forward a perspective that the current access to follow idols' information is low-cost or even zero-cost, which is easier for fans to generate para-social interactions[12]. This perspective is relatively novel. While for Bubble, the application which will be discussed in the following, sets up a money boundary for fans in receiving information. This provides a brand-new direction for para-social interaction research.

\section{THE PARA-SOCIAL INTERACTION IN BUBBLE}

\subsection{Behind-the-scene content}

Some studies believe that attraction is the radical reason of fans' positive para-social interaction behavior[8]. The reason why fans have such desire to have interaction with idols is mainly because they are attracted by the content idols present. For idols, at first, they relied on some form of role-playing, stage performances, and anything that could be called a "front stage", such as under the spotlight, in front of the camera, to show themselves and attract fans by performing or broadcast living. Nevertheless, when hidden behind the camera, away from the stage, that is, the idols' private, daily side can be called "behind the scenes"[2]. In the past, there was a clear distinction between the "front stage" and the "behind the scenes" of celebrities. Even if fans were interested in the personal lives of idols, it was difficult for them to learn about due to the lack of information receiving channels. Nowadays, with the rapid development of Internet and media technology, the public's sight gradually penetrates into the "behind the scenes", and fans can get to know portion of their idols' private life, or in other words, some privacy.

\subsubsection{Bubble in the behind-scene content}

Bubble was sold on the idea that fans could view their idols' daily life. Fans can buy tickets for designated idols and receive messages from those idols at any time. Messages can take the form of pictures like selfies, voice mails, short videos, etc., and are normally about the idols' routine life.

The paper chooses an idol with average subscriptions as an example, and uses Jack as his proxy. Figures below intuitively shows the number of messages, the percentage of the message types, and the general content of the messages sent by Jack in July, 2021 .

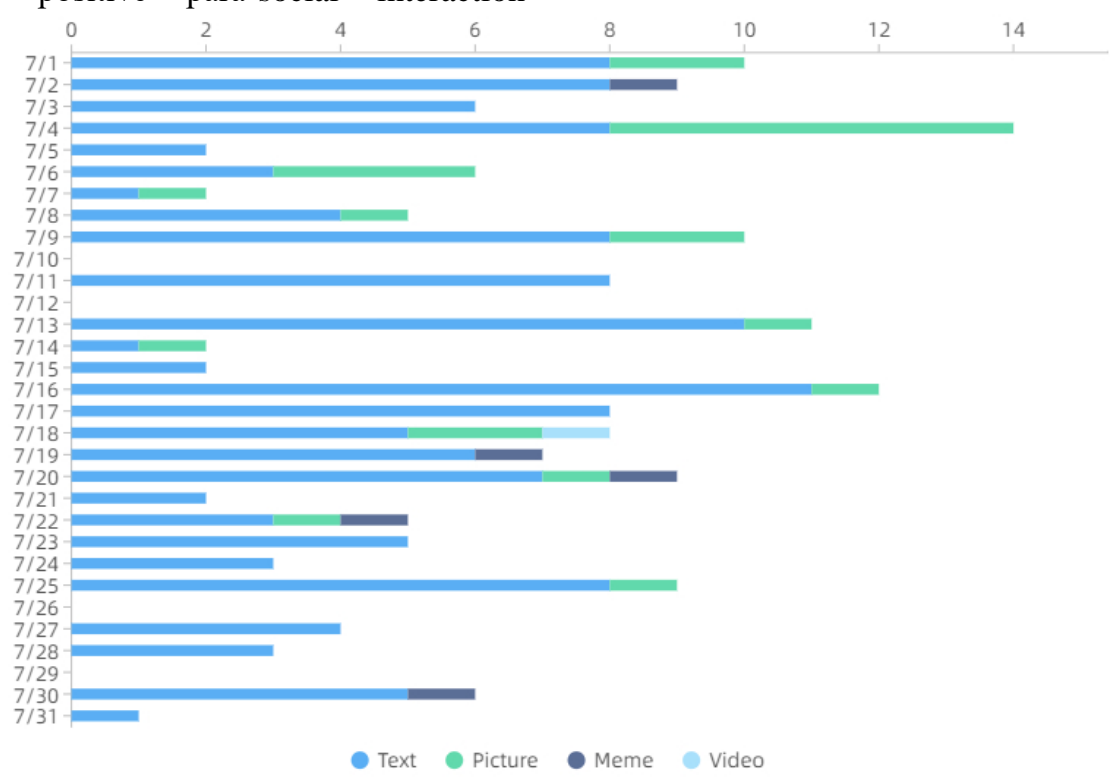

Figure 1 The number and types of messages sent by Jack on Bubble in July, 2021 


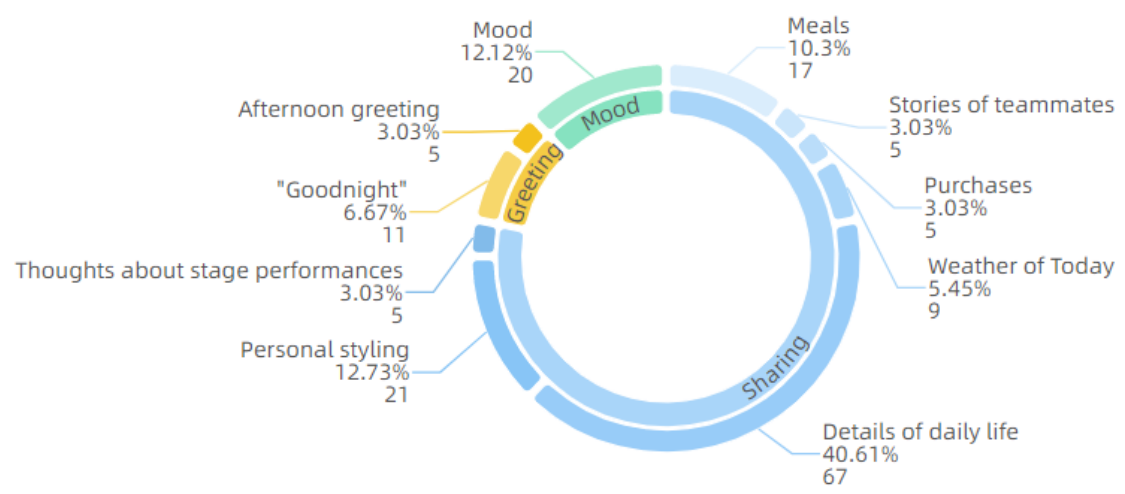

Figure 2 The general content sent by Jack on Bubble in July, 2021

As can be seen from Figure 1, Bubble provides diverse forms of messages for fans to reveal the "behind-the-scene" of idols. For Jack, he would use texts, pictures, videos and memes to deliver such contents. These various types help build the foundation for para-social interaction at the first stage. In addition, Figure 2 indicates that the majority of content in Jack's Bubble is "sharing", and the "details of daily life" accounts for $40.61 \%$ among all messages. These contents are rich in the vividness of life on the daily basis.

These fewer formal messages fulfill the requirement of fans for getting to know the "behind-the-scene" life of their idols, narrow the distance between idols and fans, and enhance the good impression of idols. Therefore, the casual, private content provides good material for building para-social interactions.

\subsection{Simulated private chatting environment}

As mentioned above, para-social interaction describes audience's imagined relationship with celebrities. By observing and interpreting celebrities' appearance, gestures, voices and behaviors, the audience feels as closely connected to the celebrities as if they were intimate friends. Traditional para-social interactions are one-way, and the relationship continues to grow as a result of constant self-disclosure by celebrities and self-interpretation by fans. With the development of social media platforms, the para-social interaction has changed from one-way to two-way to a certain extent, which is a mutual influence for both idols and fans.

In Bubble, there is a fine interfacial design to enhance this para-social interaction between idols and fans. Bubble simulates the common communication apps, and has dialogue boxes for every idol, which creates a private conversation environment and the feeling of the idol as a friend in actual.

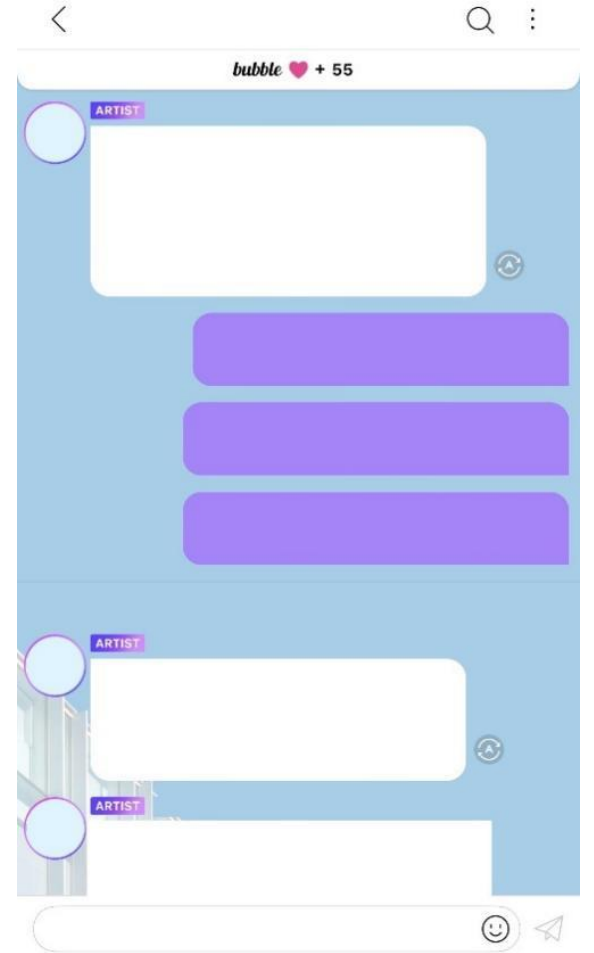

Figure 3 The chatting interface in Bubble

As can be seen from Figure 3 (what Figure 3 shows is paid content and exclusive, so in order to keep it confidential, the content is shaded), the format of interactions with idols are very similar to daily chatting with real friends. With every message sent by the idol, fans can respond with the maximum up to three texts, which dramatically improves the interactivity. At the top of the frame, a short segment "bubble +55 " indicates that the fan has been subscribing to this idol for 55 days. It is very much similar to the anniversary that friends or even lovers agree to each other, which promotes the intimacy between idols and fans. Moreover, Bubble has the sign of read/unread in front of fans' messages. This setup largely increases the motivation of fans and brings the sense of being responded. In addition, Bubble automatically replaces general terms with users' names, 
thus fans would individually feel like they are interacting with their idols on the daily basis and they know each other quite well. Bubble also embed translator in its application, as can been seen from the small " $A$ " next to the message box. This function has greatly eliminated the language barrier and improved fans' understanding of what idols have conveyed.

This private conversation environment simulated by Bubble satisfies the imagination of having close relationships with idols. Any fan who subscribes Bubble can fantasize and interpret their idol through the chatting environment with a strong sense of reality. Therefore, the one-to-one chat mode and interface design offer environment for para-social interaction.

\subsection{Establishment of membership communities}

Pop idol fans tend to exhibit great involvement with as well as emotional attachment to their idols. Fan membership can provide a sense of belonging, which to some extent foster the pervasive social bond and identification[2]. Fans can acquire membership through multiple approaches, including high participation, membership-related merchandise purchase. In result, fans would have a self-identification of being specific idol's fan, and also much similar group identity in the fan group with other fans.

In Bubble, as soon as fans pay the money for affiliating in their service, they are prohibited from the acts managed by S.M. entertainment. In other words, Bubble use adequate regulations to restrict content spreading in order to protect fan's membership. For instance, Bubble does not allow fans to "stealthily use, reproduce, sell, publish, broadcast or use of various contents (postings, sound, image, illustration, videos, etc.) distributed through services in relation to artists." Only fans that pay for their service could view the content. These regulations well establish the membership community, and fans in this community all have the same membership and self-identification. This group of fans will also spontaneously maintain the intellectual property of the content. They will take the initiative to search for relevant infringing content on the Internet, and advise those who spread exclusive content to delete the relevant posts. Fans are satisfied with being among the group with membership, constantly emphasizing the exclusivity of Bubble's message content and the pride of being fans, which enhance their loyalty to idols.

In addition, Bubble's special purchase mechanism assists in building up the internal connection among fan groups. Fans can purchase different numbers of tickets, and Bubble has different ticket sets for them. Take the boy band NCT having 16 members for example. Fans have various choices from purchasing 1 ticket (for 1 idol's message) costing $\$ 3.99$ per month, 2 tickets (for 2 idols' message) costing $\$ 6.99$ per month, 3 tickets costing $\$ 9.00$ per month. By that analogy, there is a set including 16 tickets costing $\$ 49.99$ per month, indicating that the more tickets fans purchase, the more discount they will get. Thus, this mechanism fosters fans to form a smaller community with tighter bond. If they are all NCTzen (NCT fans) while they want to receive unique content from different members of NCT, they can purchase the set of 16 tickets as group purchase and share the content in another group chat they built. These fans will then make specific rules for those sharing groups and define special nicknames and symbols in their chat rooms. Therefore, through these community-establishments, fans are inclined to strengthen their self-identification of being fans, continue to support their idols and maintain such para-social interaction.

\section{ANALYSIS ON NEW FEATURES OF PARA-SOCIAL INTERACTIONS IN BUBBLE}

\subsection{Complicated requirements for contents}

There are various types of fans pursuing different types of contents, in order to fulfill their own requirements and imagination. The essence of the difference between fans is the distinction among their desire. Some of them may suffer the loneliness and anxiety in the real world, some may seek an intimate relationship or family love, others may long for success in their career. These wrinkles in their mind enable people to take advantage of their para-social interactions with idols, constantly driving them to get the kind of content and comfort they want from idols. Since fans are with such diverse demand, there are complicated requirements for what idols send in Bubble. As Bubble has launched and been expanding, problems have aroused. The traditional para-social interaction with low cost or even zero cost, fans are more receptive in terms of content. In other words, they might not be so critical of what their idols post on social media, since they have all access to the content, for free. While the situation is rather different in Bubble, unlike the inexpensive in the previous para-social interaction, fans have a more critical eye on the messages sent by idols because they are paying for these exclusive contents, monthly. That is to say, fans will feel it is well worth the monthly payment unless they get satisfied messages in terms of quantity and quality. A Super Junior fan complained that Lee Teuk (the member of the Super Junior) sends lots of messages, which makes her feel disturbing when viewing too much notification in her phone, and the service will not be attractive unless artists are obliged to send a certain amount of messages per month. However, another fan whose favorite band member is less active than the others, commented that she paid the same money while receiving fewer messages[10]. This 
contradiction reveals that Bubble possibly needs to set regulations for all artists on how many messages should been sent, in order to avoid sending too much content, or conversely.

Not only the number of messages, fans also have various demands for the quality of them. Some may want more selfies and more voices shared, while others may only want their idols to post some trivial matters, for instance what they have had for lunch each day. It indicates that with the certain cost of purchasing messages delivered by idols, fans will have more complicated requirements for the contents they received, in terms of quantity to quality. Therefore, they do not just simply accept everything but having a more critical perspective in the para-social interaction between idols and themselves.

\subsection{Absence of public constraints - the power of cybervigilantism}

The word "cybervigilantism" was first originated around 2010, describing the phenomenon which to hunt for personal information about social deviants to restore public morality [3]. However, Jung mentioned that fan culture, especially the K-pop fandom has been criticized for those cybervifilantism-liked media culture critics and encouraging obsessive behaviors. Celebrities' basic privacy rights would be under the threat of being violated[5].

Though cybervifilantism-liked fandom caused concerns about communication in media, it also has advantages. Considering the above incidents on common social media, the para-social interactions are now kind of under public constraints. The comments, reposts, or even likes are open to every user on the social media. The antifandom of idols, for instance the online shaming, is positively supervised by their fans' cybervigilantism, in a positive way.

Moreover, as the para-social interactions between idols and fans goes further, some fans not only have special attachment to their idols, but also develop some sort of unduly accountability for telling their idols to well behave. These fans often keep their comments on their idols confidential, spreading only in a small group of like-minded people, or sending those what they called kind suggestions to idols through private messages on social media. The logic behind these behaviors is that the comment or other ways to express opinions is disclosed, and for those fans, they have concerns about others' cybervigilantism towards themselves - knowing what they say must be criticized by other fans. In order to preserve a positive image in idols' mind and avoid being reported of sending vicious messages by their own idols, these will not send those harsh words. Thus, the para-social interaction in social media, especially in fandom among anti-fans and extreme fans, is supervised by public constraints.

Nevertheless, such public constraints are almost absent from the application Bubble. The principal reason is the "stimulated private chatting environment" in Bubble. From the perspective of fans, they are having private conversations with idols, while on the screen of idols, they are in a group chat, with every single message from fans anonymous. Therefore, the concerns about malevolent responses of users have raised. Here are some worst bubble responses related to pornographic/verbal abuse to several members from the girl band, which exactly reveal the absence of public constraints in para-social interaction in Bubble.

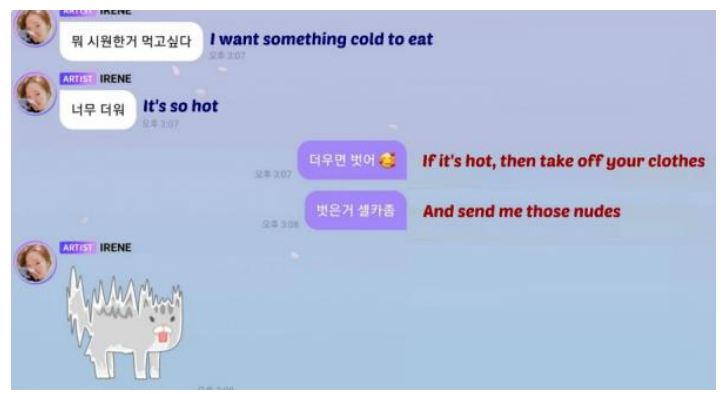

Figure 4 Irene (one of Red Velvet members)

experiencing online sexual harassment in Bubble

Seulgi, the member of Red Velvet always intends to maintain the whole team inspired and motivated. Most fans feel glad to support the whole band, while for some malevolent people, they took this opportunity as a way to shade other group members[9].

Admittedly, the private chatting environment provides good condition to stimulate and maintain the para-social interaction, but it is also notable that the absence of public constraints in Bubble, which may make those offensive behaviors hard to locate and be blocked.

\subsection{Separation between different stages of fans}

As the typical para-social interaction, star-chasing has different stages of involvement. Lynn E. McCutcheon once used the Celebrity Worship Scale to indicate the attitude of star-worshippers towards a favorite celebrity (that they themselves have named) and divided the responses into three stages[7]. The first stage is "entertainment-social", in which individuals begin to take interest to their favorite celebrities. The second stage is "intense-personal", in which individuals intertwine fantasy with reality and believe they have a special connection to their favorite stars, and idolatry has become an addictive behavior. The third stage is the most intense, in which individuals develop a "borderline-pathological" state that may even lead to risky behaviors such as committing crime. In the item "I have pictures and/or souvenirs of my favorite celebrity 
which I always keep in exactly the same place", the result loads above 40 on the intense-personal factor. It is likely that a lot of respondents interested in a particular celebrity may have one or two souvenirs, which indicates that most fans will buy some relative merchandise.

However, most of those items are tangible and useable, for instance posters, bags, keyrings, etc. While Bubble is providing intangible service, basically digital content, and under such circumstance, how many fans are willing to pay for that intangible content? For majority of fans, para-social interactions with their idols are mostly in the first and second stages. Situation gets more complex. We can have a rough conclusion that, for most fans in "intense-personal" stage, they will be the backbone of Bubble's consumers. Bubble perfectly enhances the para-social interaction between the second stage fans and their idols, but for individuals in the first stage, the mechanism of paying for digital content may block them from transforming to the second stage fans. Their love to the certain idols is not enough to support such monthly payment, and they are more willing to view the open and free content, then they are satisfied. This might not be the consequence which entertainment corporation wants because it lowers the conversion rate of fans transferring from first stage to second stage, in other words, it is the loss of potential group who are willing to spend more money, to some extent. Moreover, the certain exclusivity from spontaneously established membership communities also prevents the first stage fans from getting deeper.

\section{CONCLUSION}

In the above article, it is mentioned that how the para-social interaction is constructed in Bubble, and the new features of such para-social interaction generated in this application. Generally, the author thinks that Bubble is commercially successful in the short term, but it seems to have a relative negative impact on the para-social relationship between fans and their idols. Bubble was first launched at the beginning of the outbreak of COVID-19, when almost everyone was in a state of staying at home and in quarantine. As a result, there were fewer opportunities for people to communicate with each other, and channels for emotional acceptance and expression were cut off. Bubble may only attract existing fan groups or people with certain interest to buy services, but it cannot expand its consumers. Under the circumstance that there is already a lack of stage performances due to the pandemic, some daily sharing is also limited to paid platforms, which could have drawbacks in the long term. What's more, from idols' perspective, sharing daily life might once have been about sharing happiness with fans, but on the paid platform like Bubble, sharing has been measured.
Bubble's new interaction mode between fans and idols undoubtedly brings a new direction for the study of para-social interaction and the commercial development of the idol industry. Bubble satisfies the fans' desire for different kinds of intimate relationships, breaks through the previous barrier of two-way communication between idols and fans, and introduces the concept of purchasing information and communication with idols into para-social interaction. New features are thus flourished. After above analysis, the conclusion are as follows. First, fans are having more complicated and critical perspective in the para-social interaction. Second, the stimulated private chatting environment lacks public surveillance and leaves an open door for malevolent users. Furthermore, perhaps only second-stage fans are more willing to purchase those digital contents.

However, the research has some flaws. Firstly, not so much data related to purchasing willing can be found, it is hard to anticipate the purchasing motivation of fans. Second, more using experience is needed for analyzing.

In the next step, questionnaires will be designed to investigate how users look upon this application and how this new interaction mode and purchasing mechanism influence their behavior. More data and feedback will be collected to analyze. If possible, some similar social communication application for fans and idols will be picked out and comparative analysis will be conducted.

\section{REFERENCES}

[1] Ballantine, P. W. (2005). Effects of interactivity and product information on consumer satisfaction in an online retail setting. International Journal of Retail \& Distribution Management, 33(6), 461-471. https://doi.org/10.1108/09590550510600870

[2] Bhattacharya, C. B., Rao, H., \& Glynn, M. A. (1995). Understanding the bond of identification: An investigation of its correlates among art museum members. Journal of Marketing, 59(4), 46-57. https://doi.org/10.2307/1252327

[3] Cheong, P. H., \& Gong, J. (2010). Cyber vigilantism, transmedia collective intelligence, and civic participation. Chinese Journal of Communication, 3(4), 471-487. https://doi.org/10.1080/17544750.2010.516580

[4] Horton, D., \& Richard Wohl, R. (1956). Mass communication and para-social interaction. Psychiatry, 19(3), 215-229. https://doi.org/10.1080/00332747.1956.11023049

[5] Jung, S. (2012). Fan activism, cybervigilantism, and othering mechanisms in K-pop fandom. 
Transformative Works and Cultures, 10. https://doi.org/10.3983/twc.2012.0300

[6] Lee, J., \& Hong, I. B. (2016). Predicting positive user responses to social media advertising: The roles of emotional appeal, informativeness, and creativity. International Journal of Information Management, 36(3), 360-373. https://doi.org/10.1016/j.ijinfomgt.2016.01.001

[7] Maltby, J., Day, L., McCutcheon, L. E., Houran, J., \& Ashe, D. (2006). Extreme celebrity worship, fantasy proneness and Dissociation: Developing the measurement and understanding of celebrity worship within a clinical personality context. Personality and Individual Differences, 40(2), 273283. https://doi.org/10.1016/j.paid.2005.07.004

[8] Myers, D. G., Hou, Y., Le, G., \& Zhang, Z. (2014). Social psychology. Posts and Telecom Press.

[9] Reporter, S. (2020, June 20). Men seen harassing Red Velvet's Seulgi and Irene on bubble. KpopStarz. Retrieved October 22, 2021, from https://www.kpopstarz.com/articles/293550/20200 619/men-seen-harassing-red-velvets-seulgi-irene-b ubble.htm.

[10] Song, S. (2021, January 13). Private messages from your favorite K-pop star. The Korea Herald. Retrieved October 22, 2021, from http://m.koreaherald.com/view.php?ud=202101310 00143 .

[11] Xiao, C. (2017). An Analysis of para-social interaction in Weibo - a case study of "personality collapse" of pop stars. Today's Mass media, 25(12), 25-26.

[12] Zhu, P. (2021). Research on spread of media image of cultivating idols from the perspective of para-social interaction - A case study of TFBOYS. Southeast communication, 21(03), 113-115. 